\title{
Entanglement of Formation for a Class of Quantum States
}

\author{
Shao-Ming Fei ${ }^{\ddagger \dagger 1}$, Jürgen Jost ${ }^{\dagger 2}$, Xianqing Li-Jost ${ }^{\dagger 3}$ and Guo-Fang Wang ${ }^{\dagger 4}$ \\ $\ddagger$ Department of Mathematics, Capital Normal University, Beijing 100037 \\ ${ }^{\dagger}$ Max-Planck-Institute for Mathematics in the Sciences, 04103 Leipzig \\ $\ddagger$ Institut für Angewandte Mathematik, Universität Bonn, 53115 Bonn
}

\begin{abstract}
Entanglement of formation for a class of higher dimensional quantum mixed states is studied in terms of a generalized formula of concurrence for $N$-dimensional quantum systems. As applications, the entanglement of formation for a class of $16 \times 16$ density matrices are calculated.

PACS numbers: 03.65.Bz, 89.70.+c

Key words: Entanglement of Formation, Generalized Concurrence
\end{abstract}

Quantum entanglement plays important roles in quantum communication, information processing and quantum computing [1], such as in the investigation of quantum teleportation [2, 3, 4], dense coding [5], decoherence in quantum computers [1] and the evaluation of quantum cryptographic schemes [6]. To quantify entanglement, a well justified and mathematically tractable measure of entanglement is needed. A number of entanglement measures such as the entanglement of formation and distillation $[7,8,9]$, negativity [10, 11], and relative entropy $[9,12]$ have been proposed for bipartite states [6,8,12-17]. Nevertheless, most

\footnotetext{
${ }^{1}$ Email: fei@uni-bonn.de

${ }^{2}$ Email: jjost@mis.mpg.de

${ }^{3}$ Email: Xianqing.Li-Jost@mis.mpg.de

${ }^{4}$ Email: Guofang.Wang@mis.mpg.de
} 
proposed measures of entanglement involve extremizations which are difficult to handle analytically.

The "entanglement of formation" is intended to quantify the amount of quantum communication required to create a given state [7]. Although it is defined for arbitrary dimensions, so far no explicit analytic formulae for entanglement of formation have been found for systems larger than a pair of qubits, due to the fact that two dimensional bipartite mixed states are special in many ways [16], except for some special symmetric states [17].

In this letter we study the entanglement of formation for a class of higher dimensional quantum mixed states. For certain $N$-dimensional pure quantum systems, we show that the entanglement of formation is a monotonically increasing function of a kind of generalized concurrence. As applications, the entanglement of formation for a class of $16 \times 16$ density matrices is calculated in detail. The method applies to a large class of quantum states. The construction of these states are presented for $N$ dimensional, $N=2^{k+1}, 2 \leq k \in \mathbb{N}$, bipartite systems.

Let $\mathcal{H}$ be an $N$-dimensional complex Hilbert space with orthonormal basis $e_{i}, i=1, \ldots, N$. A pure state on $\mathcal{H} \otimes \mathcal{H}$ is generally of the form,

$$
|\psi\rangle=\sum_{i, j=1}^{N} a_{i j} e_{i} \otimes e_{j}, \quad a_{i j} \in \mathbb{C}
$$

with normalization

$$
\sum_{i, j=1}^{N} a_{i j} a_{i j}^{*}=1
$$

The entanglement of formation $E$ is defined as the entropy of either of the two sub-Hilbert space $\mathcal{H} \otimes \mathcal{H}[8]$,

$$
E(|\psi\rangle)=-\operatorname{Tr}\left(\rho_{1} \log _{2} \rho_{1}\right)=-\operatorname{Tr}\left(\rho_{2} \log _{2} \rho_{2}\right)
$$

where $\rho_{1}$ (resp. $\rho_{2}$ ) is the partial trace of $|\psi\rangle\langle\psi|$ over the first (resp. second) Hilbert space of $\mathcal{H} \otimes \mathcal{H}$.

Let $A$ denote the matrix with entries given by $a_{i j}$ in (1). $\rho_{1}$ can be expressed as

$$
\rho_{1}=A A^{\dagger}
$$

For a given density matrix of a pair of quantum systems on $\mathcal{H} \otimes \mathcal{H}$, consider all possible purestate decompositions of $\rho$, i.e., all ensembles of state $\left|\psi_{i}\right\rangle$ of the form (1) with probabilities 
$p_{i}$,

$$
\rho=\sum_{i=1}^{M} p_{i}\left|\psi_{i}\right\rangle\left\langle\psi_{i}\right|, \quad \sum_{i=1}^{M} p_{i}=1
$$

for some $M \in \mathbb{N}$. The entanglement of formation for the mixed state $\rho$ is defined as the average entanglement of the pure states of the decomposition, minimized over all possible decompositions of $\rho$,

$$
E(\rho)=\min \sum_{i=1}^{M} p_{i} E\left(\left|\psi_{i}\right\rangle\right) .
$$

It is a challenge to calculate (5) for general $N$. Till now a general explicit formula of $E(\rho)$ is obtained only for the case $N=2$. In this case (3) can be written as

$$
\left.E(|\psi\rangle)\right|_{N=2}=h\left(\frac{1+\sqrt{1-C^{2}}}{2}\right),
$$

where

$$
h(x)=-x \log _{2} x-(1-x) \log _{2}(1-x),
$$

$C$ is called concurrence [15]:

$$
C(|\psi\rangle)=|\langle\psi \mid \tilde{\psi}\rangle|=2\left|a_{11} a_{22}-a_{12} a_{21}\right|
$$

where $|\tilde{\psi}\rangle=\sigma_{y} \otimes \sigma_{y}\left|\psi^{*}\right\rangle,\left|\psi^{*}\right\rangle$ is the complex conjugate of $|\psi\rangle, \sigma_{y}$ is the Pauli matrix, $\sigma_{y}=\left(\begin{array}{cc}0 & -i \\ i & 0\end{array}\right)$.

As $E$ is a monotonically increasing function of $C, C$ can be also taken as a kind of measure of entanglement. Calculating (5) is reduced to calculate the corresponding minimum of $C(\rho)=\min \sum_{i=1}^{M} p_{i} C\left(\left|\psi_{i}\right\rangle\right)$, which simplifies the problems.

For $N \geq 3$, there is no such concurrence $C$ in general. The concurrences discussed in [18] can be only used to judge whether a pure state is separable (or maximally entangled) or not $[19,20]$. The entanglement of formation is no longer a monotonically increasing function of these concurrences. Nevertheless, for a special class of quantum states, we can find certain quantities (generalized concurrence) to simplify the calculation of the corresponding entanglement of formation.

[Theorem 1]. If $A A^{\dagger}$ has only two non-zero eigenvalues (each of which may be degenerate), the maximal non-zero diagonal determinant $D$ of $A A^{\dagger}$ is a generalized concurrence. The entanglement of formation of the corresponding pure state is a monotonically increasing function of $D$. 
[Proof]. Let $\lambda_{1}$ (resp. $\lambda_{2}$ ) be the two non-zero eigenvalues of $A A^{\dagger}$ with degeneracy $n$ (resp. $m), n+m \leq N$. That is,

$$
D=\lambda_{1}^{n} \lambda_{2}^{m}
$$

From the normalization of $|\psi\rangle$, one has $\operatorname{Tr}\left(A A^{\dagger}\right)=1$, i.e.,

$$
n \lambda_{1}+m \lambda_{2}=1
$$

$\lambda_{1}\left(\right.$ resp. $\left.\lambda_{2}\right)$ takes values $\left(0, \frac{1}{n}\right)$ (resp. $\left.\left(0, \frac{1}{m}\right)\right)$. In this case the entanglement of formation of $|\psi\rangle$ is given by

$$
E(|\psi\rangle)=-n \lambda_{1} \log _{2} \lambda_{1}-m \lambda_{2} \log _{2} \lambda_{2}
$$

According to (6) and (7) we get

$$
\frac{\partial E}{\partial D}=\frac{m \lambda_{1}^{1-n}}{1-n \lambda_{1}-m \lambda_{1}}\left(\frac{1-n \lambda_{1}}{m}\right)^{1-m} \log _{2} \frac{1-n \lambda_{1}}{m \lambda_{1}},
$$

which is positive for $\lambda_{1} \in\left(0, \frac{1}{n}\right)$. Therefore $E(|\psi\rangle)$ is a monotonically increasing function of $D$. $D$ is a generalized concurrence and can be taken as a kind of measure of entanglement in this case.

Remark: We have assumed that $\lambda_{1}, \lambda_{2} \neq 0$ in our theorem. In fact the right hand side of (9) keeps positive even when $\lambda_{1}$ (or equivalently $\lambda_{2}$ ) goes to zero. Hence $E(|\psi\rangle$ ) is a monotonically increasing function of $D$ for $\lambda_{1} \in\left[0, \frac{1}{n}\right]$ (resp. $\lambda_{2} \in\left[0, \frac{1}{m}\right]$ ) satisfying the relation (7). Nevertheless if $\lambda_{1}=0$ (or $\lambda_{2}=0$ ), from (6) one gets $D=0$, which does not necessarily mean that the corresponding state $|\psi\rangle$ is separable. As $E(|\psi\rangle)$ is just a monotonically increasing function of $D, D$ only characterizes the relative degree of the entanglement among the class of these states.

From (7) and (8), the quantum states with the measure of entanglement characterized by $D$ are generally entangled. They are separated when $n=1, \lambda_{1} \rightarrow 1\left(\lambda_{2} \rightarrow 0\right)$ or $m=1$, $\lambda_{2} \rightarrow 1\left(\lambda_{1} \rightarrow 0\right)$. For the case $n=m>1$, all the pure states in this class are non-separable. In this case, we have

$$
E(|\psi\rangle)=n\left(-x \log _{2} x-\left(\frac{1}{n}-x\right) \log _{2}\left(\frac{1}{n}-x\right)\right)
$$

where

$$
x=\frac{1}{2}\left(\frac{1}{n}+\sqrt{\frac{1}{n^{2}}\left(1-d^{2}\right)}\right)
$$


and

$$
d \equiv 2 n D^{\frac{1}{2 n}}=2 n \sqrt{\lambda_{1} \lambda_{2}} .
$$

In this case we define $d$ to be the generalized concurrence. $d$ takes value from 0 to 1 . From (10) one can show that $E(d)$ is a convex function (that is, curving upward):

$$
\frac{\partial^{2} E}{\partial d^{2}}=\frac{\log \frac{1+\sqrt{1-d^{2}}}{1-\sqrt{1-d^{2}}}-2 \sqrt{1-d^{2}}}{\left(1-d^{2}\right)^{3 / 2} \log 4}>0, \quad \forall d \in[0,1] .
$$

Instead of calculating $E(\rho)$ directly, one may calculate the minimum decomposition of $D(\rho)$ or $d(\rho)$ to simplify the calculations. In the following, as an example, we calculate the entanglement of formation for a class of mixed states with $N=4$.

We consider a class of pure states (1) with the matrix $A$ given by

$$
A=\left(\begin{array}{cccc}
0 & b & a_{1} & b_{1} \\
-b & 0 & c_{1} & d_{1} \\
a_{1} & c_{1} & 0 & -e \\
b_{1} & d_{1} & e & 0
\end{array}\right),
$$

$a_{1}, b_{1}, c_{1}, d_{1}, b, e \in \mathbb{C}$. The matrix $A A^{\dagger}$ has two eigenvalues with degeneracy two, i.e., $n=m=2$.

$$
\operatorname{det}\left(A A^{\dagger}\right)=\left|b_{1} c_{1}-a_{1} d_{1}+b e\right|^{4} .
$$

According to our theorem, the generalized concurrence

$$
d=4\left|b_{1} c_{1}-a_{1} d_{1}+b e\right|
$$

is a kind of measure of entanglement for all pure states of the form (12).

Let $p$ be a $16 \times 16$ matrix with only non-zero entries $p_{1,16}=p_{2,15}=-p_{3,14}=p_{4,10}=$ $p_{5,12}=p_{6,11}=p_{7,13}=-p_{8,8}=-p_{9,9}=p_{10,4}=p_{11,6}=p_{12,5}=p_{13,7}=-p_{14,3}=p_{15,2}=p_{16,1}=$ 1. $d$ in (14) can be written as

$$
d=\left|\left\langle\psi \mid p \psi^{*}\right\rangle\right| \equiv|\langle\langle\psi \mid \psi\rangle\rangle|,
$$

where $\langle\langle\psi \mid \psi\rangle\rangle=\left\langle\psi \mid p \psi^{*}\right\rangle$.

We now calculate the entanglement of formation for a special class of mixed states. Let $\Psi$ denote the set of pure states (1) with $A$ given as the form of (12). We consider all mixed states with density matrix $\rho$ such that its decompositions are of the form

$$
\rho=\sum_{i=1}^{M} p_{i}\left|\psi_{i}\right\rangle\left\langle\psi_{i}\left|, \quad \sum_{i=1}^{M} p_{i}=1, \quad\right| \psi_{i}\right\rangle \in \Psi .
$$


Let $s \leq 16$ be the rank of $\rho$ and $\left|v_{i}\right\rangle, i=1, \ldots, s$, be a complete set of orthogonal eigenvectors corresponding to the nonzero eigenvalues of $\rho$, such that $\left\langle v_{i} \mid v_{i}\right\rangle$ is equal to the $i$ th eigenvalue. Other decomposition $\left\{\left|w_{i}\right\rangle\right\}$ of $\rho$ can then be obtained through unitary transformations:

$$
\left|w_{i}\right\rangle=\sum_{j=1}^{s} U_{i j}^{*}\left|v_{j}\right\rangle,
$$

where $U$ is a $t \times t$ unitary matrix, $t \geq s$. The states $\left|w_{i}\right\rangle$ are so normalized that $\rho=$ $\sum_{i}\left|w_{i}\right\rangle\left\langle w_{i}\right|$. It is obvious that for any $\left|\psi_{i}\right\rangle \in \Psi$, the state of complex linear combination of $\left|\psi_{i}\right\rangle$ (unitary transformations) also belongs to $\Psi$.

The decomposition according to the orthogonal eigenvectors $\left|v_{i}\right\rangle$ of $\rho$ is not the one satisfying (5) in general. As the generalized concurrence can be written as the form (15) for a pure state, we consider the quantity $\left\langle\left\langle w_{i} \mid w_{j}\right\rangle\right\rangle$. From (17) we have

$$
\left\langle\left\langle w_{i} \mid w_{j}\right\rangle\right\rangle=\left(U \tau U^{T}\right)_{i j}
$$

where the matrix $\tau$ is defined by $\tau_{i j} \equiv\left\langle\left\langle v_{i} \mid v_{j}\right\rangle\right\rangle$. The matrix $p$ in (15) is a symmetric one. Therefore $\tau$ is also symmetric and can always be diagonalized by a unitary matrix $U$ such that $U \tau U^{T}=\operatorname{diag}\left(\Lambda_{1}, \ldots, \Lambda_{s}\right)[21]$. The diagonal elements $\Lambda_{i}$, in deceasing order, can always be made to be real and non-negative. Since $U \tau \tau^{*} U^{\dagger}$ is also diagonal, $\Lambda_{i}$ are just the square roots of the eigenvalues of $\tau \tau^{*}$. It is straightforward to check that they are also the eigenvalues of the Hermitian matrix $R \equiv \sqrt{\sqrt{\rho} p \rho^{*} p \sqrt{\rho}}$, or, alternatively, the square roots of the eigenvalues of the non-Hermitian matrix $\rho p \rho^{*} p$.

Hence there always exits a decomposition consisting of states $\left|w_{i}\right\rangle, i=1, \ldots, s$, such that

$$
\left\langle\left\langle w_{i} \mid w_{j}\right\rangle\right\rangle=\Lambda_{i} \delta_{i j}
$$

We can now deal with the problem in a way similar to [15]. Set

$$
\left|y_{1}\right\rangle=\left|w_{1}\right\rangle, \quad\left|y_{j}\right\rangle=i\left|w_{j}\right\rangle \quad \text { for } j=2, \ldots, s .
$$

Any decomposition can be written in terms of the states $\left|y_{i}\right\rangle$ via the equation

$$
\left|z_{i}\right\rangle=\sum_{j=1}^{s} V_{i j}^{*}\left|y_{j}\right\rangle,
$$

where $V$ is a $t \times s$ matrix whose $s$ columns are othonormal vectors. 
The average concurrence of a general decomposition is given by

$$
\langle d\rangle=\sum_{i}\left|\left(V Y V^{T}\right)_{i i}\right|=\sum_{i}\left|\sum_{j}\left(V_{i j}\right)^{2} Y_{j j}\right|
$$

where $Y$ is the real diagonal matrix defined by $Y_{i j}=\left\langle\left\langle y_{i} \mid y_{j}\right\rangle\right\rangle$. Using the fact that $\sum_{i}\left|\left(V_{i j}\right)^{2}\right|=1$, one gets

$$
\langle d\rangle \geq\left|\sum_{i j}\left(V_{i j}\right)^{2} Y_{j j}\right| \geq \Lambda_{1}-\sum_{i=2}^{16} \Lambda_{i} .
$$

Therefore the minimum decomposition of the generalized concurrence is

$$
d(\rho)=\Lambda_{1}-\sum_{i=2}^{16} \Lambda_{i}
$$

Similar to the case $N=2$, there are decompositions such that the generalized concurrence of each individual state is equal to $d(\rho)$. Therefore the average entanglement is $E(d(\rho))$.

Different from the case $N=2$, the entanglement of formation of density matrices (16) can not be zero in general. As every individual pure state in the decompositions is generally an entangled one, this class of mixed states are not separable.

In the following we call an $N$-dimensional pure state (1) $d$-computable if $A$ satisfies the following relations:

$$
\begin{aligned}
& \operatorname{det}\left(A A^{\dagger}\right)=\left([A][A]^{*}\right)^{N / 2} \\
& \operatorname{det}\left(A A^{\dagger}-\lambda I d_{N}\right)=\left(\lambda^{2}-\|A\| \lambda+[A][A]^{*}\right)^{N / 2},
\end{aligned}
$$

where $[A]$ and $\|A\|$ are any quadratic forms of $a_{i j}$ (these quadratic forms could be different for different matrix $A$ ), $I d_{N}$ is the $N \times N$ identity matrix. We denote $\mathcal{A}$ the set of matrices satisfying (22), which implies that for $A \in \mathcal{A}, A A^{\dagger}$ has at most two different eigenvalues and each one has order $N / 2$. Formula (21) can be generalized to general $N^{2} \times N^{2}$ density matrices with decompositions on $N$-dimensional $d$-computable pure states.

A class of $N$-dimensional, $N=2^{k}, 2 \leq k \in \mathbb{N}, d$-computable states has been constructed in [22]. These states give rise to a special class of density matrices with decompositions in these pure states, and the entanglement of formation for these density matrices can be calculated analytically according to the method above.

Let $A$ be an $N \times N$ matrix with entries $a_{i j} \in \mathbb{C}, i, j=1, \ldots, N$, with the following properties: 
Set

$$
A_{2}=\left(\begin{array}{cc}
a & -c \\
c & d
\end{array}\right)
$$

where $a, c, d \in \mathbb{C}$. For any $b_{1}, c_{1} \in \mathbb{C}$, a $4 \times 4$ matrix $A_{4} \in \mathcal{A}$ can be constructed in the following way,

$$
A_{4}=\left(\begin{array}{cc}
B_{2} & A_{2} \\
-A_{2}^{t} & C_{2}^{t}
\end{array}\right)=\left(\begin{array}{cccc}
0 & b_{1} & a & -c \\
-b_{1} & 0 & c & d \\
-a & -c & 0 & -c_{1} \\
c & -d & c_{1} & 0
\end{array}\right)
$$

where

$$
B_{2}=b_{1} J_{2}, \quad C_{2}=c_{1} J_{2}, \quad J_{2}=\left(\begin{array}{cc}
0 & 1 \\
-1 & 0
\end{array}\right) .
$$

$A_{4}$ satisfies the relations in (22):

$$
\begin{aligned}
&\left|A_{4} A_{4}^{\dagger}\right|=\left[\left(b_{1} c_{1}+a d+c^{2}\right)\left(b_{1} c_{1}+a d+c^{2}\right)^{*}\right]^{2}=\left(\left[A_{4}\right]\left[A_{4}\right]^{*}\right)^{2}, \\
&\left|A_{4} A_{4}^{\dagger}-\lambda I d_{4}\right|=\left(\lambda^{2}-\left(b_{1} b_{1}^{*}+c_{1} c_{1}^{*}+a a^{*}+2 c c^{*}+d d^{*}\right) \lambda\right. \\
&\left.+\left(b_{1} c_{1}+a d+c^{2}\right)\left(b_{1} c_{1}+a d+c^{2}\right)^{*}\right)^{2} \\
&=\left(\lambda^{2}-\left\|A_{4}\right\| \lambda+\left[A_{4}\right]\left[A_{4}\right]^{*}\right)^{2}
\end{aligned}
$$

where

$$
\left[A_{4}\right]=\left(b_{1} c_{1}+a d+c^{2}\right), \quad\left\|A_{4}\right\|=b_{1} b_{1}^{*}+c_{1} c_{1}^{*}+a a^{*}+2 c c^{*}+d d^{*}
$$

$A_{8} \in \mathcal{A}$ can be obtained from $A_{4}$,

$$
A_{8}=\left(\begin{array}{cc}
B_{4} & A_{4} \\
-A_{4}^{t} & C_{4}^{t}
\end{array}\right)
$$

where

$$
B_{4}=b_{2} J_{4}, \quad C_{4}=c_{2} J_{4}, \quad J_{4}=\left(\begin{array}{cccc}
0 & 0 & 0 & 1 \\
0 & 0 & 1 & 0 \\
0 & -1 & 0 & 0 \\
-1 & 0 & 0 & 0
\end{array}\right), \quad b_{2}, c_{2} \in \mathbb{C} \text {. }
$$

For general construction of high dimensional matrices $A_{2^{k+1}} \in \mathcal{A}, 2 \leq k \in \mathbb{N}$, we have

$$
A_{2^{k+1}}=\left(\begin{array}{cc}
B_{2^{k}} & A_{2^{k}} \\
(-1)^{\frac{k(k+1)}{2}} A_{2^{k}}^{t} & C_{2^{k}}^{t}
\end{array}\right) \equiv\left(\begin{array}{cc}
b_{k} J_{2^{k}} & A_{2^{k}} \\
(-1)^{\frac{k(k+1)}{2}} A_{2^{k}}^{t} & c_{k} J_{2^{k}}^{t}
\end{array}\right),
$$




$$
J_{2^{k+1}}=\left(\begin{array}{cc}
0 & J_{2^{k}} \\
(-1)^{\frac{(k+1)(k+2)}{2}} J_{2^{k}}^{t} & 0
\end{array}\right),
$$

where $b_{k}, c_{k} \in \mathbb{C}, B_{2^{k}}=b_{k} J_{2^{k}}, C_{2^{k}}=c_{k} J_{2^{k}}$. It can be verified that $A_{2^{k}}$ satisfies the following relations $[22]$ :

$$
\begin{aligned}
& \left|A_{2^{k+1}} A_{2^{k+1}}^{\dagger}\right|=\left(\left[A_{2^{k+1}}\right]\left[A_{2^{k+1}}\right]^{*}\right)^{2^{k}}=\left[\left((-1)^{\frac{k(k+1)}{2}} b_{k} c_{k}-\left[A_{2^{k}}\right]\right)\left((-1)^{\frac{k(k+1)}{2}} b_{k}^{*} c_{k}^{*}-\left[A_{2^{k}}\right]^{*}\right)\right]^{2^{k}}, \\
& \left|A_{2^{k+1}} A_{2^{k+1}}^{\dagger}-\lambda I d_{2^{k+1}}\right|=\left(\lambda^{2}-\| A_{2^{k+1}}|| \lambda+\left[A_{2^{k+1}}\right]\left[A_{2^{k+1}}\right]^{*}\right)^{2^{k}} .
\end{aligned}
$$

Therefore the states given by (27) are $d$-computable. In terms of (11) the generalized concurrence for these states is given by

$$
d_{2^{k+1}}=2^{k+1}\left|\left[A_{2^{k+1}}\right]\right|=2^{k+1}\left|b_{k} c_{k}+b_{k-1} c_{k-1}+\ldots+b_{1} c_{1}+a d+c^{2}\right| .
$$

Let $p_{2^{k+1}}$ be a symmetric anti-diagonal $2^{2 k+2} \times 2^{2 k+2}$ matrix with all the anti-diagonal elements 1 except for those at rows $2^{k+1}-1+s\left(2^{k+2}-2\right), 2^{k+1}+s\left(2^{k+2}-2\right), 2^{k+2}-1+$ $s\left(2^{k+2}-2\right), 2^{k+2}+s\left(2^{k+2}-2\right), s=0, \ldots, 2^{k+1}-1$, which are $-1 . d_{2^{k+1}}$ can be written as

$$
d_{2^{k+1}}=\left|\left\langle\psi_{2^{k+1}} \mid p_{2^{k+1}} \psi_{2^{k+1}}^{*}\right\rangle\right| \equiv\left|\left\langle\left\langle\psi_{2^{k+1}} \mid \psi_{2^{k+1}}\right\rangle\right\rangle\right|,
$$

where

$$
\left|\psi_{2^{k+1}}\right\rangle=\sum_{i, j=1}^{2^{k+1}}\left(A_{2^{k+1}}\right)_{i j} e_{i} \otimes e_{j} .
$$

According to the calculations on entanglement of formation for $d$-computable states, for a $2^{2 k+2} \times 2^{2 k+2}$ density matrix $\rho_{2^{k+2}}$ with decompositions on pure states of the form (31), its entanglement of formation is given by $E\left(d_{2^{k+1}}\left(\rho_{2^{2 k+2}}\right)\right)$, where

$$
d_{2^{k+1}}\left(\rho_{2^{2 k+2}}\right)=\Omega_{1}-\sum_{i=2}^{2^{2 k+2}} \Omega_{i},
$$

and $\Omega_{i}$, in decreasing order, are the the square roots of the eigenvalues of the matrix $\rho_{2^{2 k+2}} p_{2^{k+1}} \rho_{2^{2 k+2}}^{*} p_{2^{k+1}}$.

We have studied the entanglement of formation for a class of higher dimensional quantum mixed states. It is shown that for certain $N$-dimensional pure quantum systems, the entanglement of formation is a monotonically increasing function of a generalized concurrence. From this generalized concurrence the entanglement of formation for a large class of quantum states can be calculated analytically. The physical properties of these states are remained to be studied further. 


\section{References}

[1] See, for example, D.P. DiVincenzo, Science 270, 255 (1995).

[2] C.H. Bennett, G. Brassard, C. Crépeau, R. Jozsa, A. Peres, and W.K. Wootters, Phys. Rev. Lett. 70, 1895 (1993).

[3] S. Albeverio and S.M. Fei, Phys. Lett. A 276, 8-11(2000).

[4] S. Albeverio and S.M. Fei and W.L. Yang, Commun. Theor. Phys. 38, 301-304(2002); Phys. Rev. A 66, 012301(2002).

[5] C.H. Bennett and S.J. Wiesner, Phys. Rev. Lett. 69, 2881 (1992).

[6] See, for example, C.A. Fuchs, N. Gisin, R.B. Griffiths, C-S. Niu, and A. Peres, Phys. Rev., A 56, 1163 (1997) and references therein.

[7] C.H. Bennett, D.P. DiVincenzo, J.A. Smolin, and W.K. Wootters, Phys. Rev. A 54, 3824 (1996).

[8] C.H. Bennett, H.J. Bernstein, S. Popescu, and B. Schumacher, Phys. Rev. A 53, 2046 (1996).

[9] V. Vedral, M.B. Plenio, M.A. Rippin, and P.L. Knight, Phys. Rev. Lett. 78, 2275 (1997);

V. Vedral, M.B. Plenio, K. Jacobs, and P.L. Knight, Phys. Rev. A 56, 4452 (1997);

V. Vedral and M.B. Plenio, Phys. Rev. A 57, 1619 (1998).

[10] A. Peres, Phys. Rev. Lett. 771413 (1996).

[11] K. Życzkowski and P. Horodecki, Phys. Rev. A 58, 883 (1998).

[12] B. Schumacher and M.D. Westmoreland, Relative entropy in quantum information theory, quant-ph/0004045.

[13] M. Horodecki, P. Horodecki, and R. Horodecki, Phys. Rev. Lett. 80, 5239 (1998).

[14] E.M. Rains, IEEE Trans. Inform. Theory 47, 2921-2933 (2001). 
[15] S. Hill and W.K. Wootters, Phys. Rev. Lett. 78, 5022 (1997).

W.K. Wootters, Phys. Rev. Lett. 80, 2245 (1998).

[16] R.F. Werner and M.M. Wolf, Phys. Rev. A 61, 062102 (2000).

[17] B.M. Terhal, K. Gerd and K.G.H. Vollbrecht, Phys. Rev. Lett. 85, 2625 (2000).

[18] A.Uhlmann, Phys. Rev. A 62, 032307 (2000).

S. Albererio and S.M. Fei, J. Opt. B: Quantum Semiclass. Opt. 3 1-5(2001).

P. Rungta, V. Bužek, C.M. Caves, M. Hillery, G.J. Milburn, Phys. Rev. A 64, (042315) (2001).

[19] S. Albeverio, S.M. Fei and D. Goswami, Phys. Lett. A, 91-96 (2001).

[20] S.M. Fei, X.H. Gao, X.H. Wang, Z.X. Wang and K. Wu, Phys. Lett. A 300, 559$566(2002)$.

[21] R.A. Horn and C.R. Johnson, Matrix Analysis, Cambridge University Press, New York, 1985.

[22] S.M. Fei and X.Q. Li, A Special Class of Matrices and Quantum Entanglement, MISpreprint 2002. 